\title{
Aspleniaceae and Polypodiaceae from the coastal regions of Riau, Indonesia and their palynological study
}

\author{
Nery Sofiyanti* \\ Department of Biology, Faculty of Math and Natural Sciences, \\ Universitas Riau, Kampus Bina Widya, Panam, Pekanbaru, Riau, Indonesia \\ *Corresponding Author: nery.sofiyanti@ lecturer.unri.ac.id \\ [Accepted: 26 August 2019]

\begin{abstract}
The fern inventorization in the coastal area of Riau, Indonesia have never been reported. This study aimed to identify Aspleniaceae and Polypodiaceae species from the coastal region of Riau and examined their morphology and palynology. Samples were collected from the field using exploration method and were then prepared for palynologycal study. Spore observation was carried out using Scanning Electron Microcopy. A total of 7 species were identified in this study (3 Aspleniaceae species and 4 Polypodiaceae species), with 2 species are new record in the peridoflora checklist from Riau, i.e. Asplenium longissimum and Asplenium glaucophyllum. We observed monolete spore from all of the examined species.
\end{abstract}

Keywords: Paku - Riau pesisir - Pteridophyte - Spore.

[Cite as: Sofiyanti N (2019) Aspleniaceae and Polypodiaceae from the coastal regions of Riau, Indonesia and their palynological study. Tropical Plant Research 6(2): 326-334]

\section{INTRODUCTION}

Aspleniaceae L. (Spleenwort) (Smith et al. 2006a) and Polypodiaceae Bercht. \& J. S. Presl. are fern families within Order Polypodiales, Class Polypodiopsida (Smith et al. 2006a, Lashin 2012, Silva \& Schwartsburd 2017). The members of Aspleniaceae are characterized by their monomorphic blade (Smith et al. 2006b), linear sori with a flap-like indusium along the margin (Lashin 2012) and comprises approximately 750 species worldwide (Smith et al. 2006a, Ohlsen et al. 2014). While Polypodiaceae has creeping rhizome, monomorphic or dimorphic blade (Smith et al. 2006b) and commonly abaxial roundish or elongated sori without indusium (Silva \& Schwartsburd 2017). This family consists of $c a$. 50 genera and 1.200 species (Smith et al. 2006a).

The record of pteridophyte checklist in Riau, Sumatera Island, Indonesia had been reported by Sofiyanti (2013), Sofiyanti et al. (2015a,b), Marpaung et al. (2016), Yusna et al. (2016), Wulandari et al. (2016) and Sofiyanti et al. (2016). While the spore morphology of fern from Riau only reported on members of Family Pteridaceae (Marpaung et al. 2016). Up to the present, there is no record had been reported on fern study from the coastal regions of Riau Province that located on the eastern part of Riau. The districts in this coastal region, also consisted of many islands such as Halang, Rupat, Selat Panjang, and Rangsang Island that facing the Malaca Strait. During our field works at these areas in 2017, we observed the occurrence of Aspleniaceae and Polypodiaceae species. The aim of this study was to report members of both families, as well as examined their morphology and palynology.

\section{MATERIALS AND METHODS}

Samples collection

We carried out explorations on 4 districts located in coastal regions of Riau Province, Indonesia, i.e. Rokan Hilir, Dumai, Siak and Kepulauan Meranti. The specimens were collected from the fields and then photographed before being prepared for the herbarium. The synonym was checked using theplantlist.org. All of the specimens were deposited in Herbarium Riauensis, Department of Biology, Faculty of Math and Natural Science, Universitas Riau. The morphological characterizations followed Piggott \& Piggott (1988) and Sofiyanti et al. (2015a). 
Palynological study

Spore specimens were coated using gold (Au) based on Regalado \& Saânche (2002). The coating process was conducted without any pre-treatment following Regalado \& Saânche (2002) and Moran et al. (2007). The specimens were then observed using ZEISS Scanning Electron Microscope EVO MA 10 (PT. Vanadia). The measurement of spore size was based on 15 grains. The determination of spore characteristic followed the definition of Erdtman (1957), Nayar \& Devi (1964), Pal \& Pal (1970), Regalado \& Sanchez (2002), Lashin (2012) and Wei \& Dong (2012).

\section{RESULTS AND DISCUSSION}

Taxonomic Treatment

\section{ASPLENIACEAE}

Asplenium glaucophyllum Alderw. Bull. Jard. Bot. Buitenzorg Ser. 2, VII. 6. 1912.

(Figs. 1A-C)

Uncommon. Epiphyte on Oil Palm tree. Rhizome creeping, black, hairy. Stipe and rachis dull, black. Laminae bipinnatifid, $c a .85 \mathrm{~cm}$. Pinnule with several lobes. Sorus linear almost curved toward margin of pinnule.

Note: We only found this species in one region (Siak), as epiphytic fern on Oil Palm tree (Elaeis guineensis Jack, Arecaceae). Asplenium glaucophyllum is abundant at the tree base. No record had been reported on this species in Riau, Sumatera Island. Beukema \& van Noordwijk (2004) had reported the occurrence of this species in Sumatera island, from Jambi Province.

Specimens: INDONESIA, Riau, Siak, Koto Gasip, Elevation $52 \mathrm{~m}, \mathrm{~N} 01^{\circ} 00^{\prime} 32.2^{\prime \prime} \mathrm{E} 102^{\circ} 42^{\prime} 38.5^{\prime \prime}$, July $^{\text {th }}$ 2017, Nery Sofiyanti, AGL1, AGL2, AGL3 (HR).

Asplenium longissimum Blume. Enum. Pl. Javae 2: 1781828.

(Figs. 1D-F)

Common. Terrestrial. Rhizome short, scaly. Stipes $c a .25 \mathrm{~cm}$ or more, purplish black. Laminae elongated, ca. $1 \mathrm{~m}$ long or more, consisted of up to 50 pairs of pinna, bulbil present near apex. Pinnae dark green, slightly serrate, gradually narrowing toward apex. Sori linear, little curved.

Note: This species is commonly found on shady place or under the tree, especially on moist habitat. No record had been reported in Riau pteridoflora checklist.

Specimens: INDONESIA, Riau, Bengkalis, Elevation $5 \mathrm{~m}, \mathrm{~N} \mathrm{00} 40^{\prime} 35.5^{\prime \prime}$ E $101^{\circ} 48^{\prime} 05.3^{\prime \prime}$, July $6^{\text {th }} 2017$, Nery Sofiyanti, ALO1, ALO2, ALO3, ALO4 (HR).

Asplenium nidus L. Sp. Pl. 2: 10791753.

(Figs. 1G-I)

Asplenium australasicum (J. Sm.) Hook. Fil. Exot. t. 881854.

Asplenium ficifolium Goldm. Nov. Act. 19: 4611843.

Neottopteris australasica J. Sm. Cult. Ferns 491857.

Neottopteris mauritiana Fée. Mem. Foug., Gen. Filic. 2031850.

Common. Epiphytic, sometimes lithophytic. Rhizome short, dark brown, scally. Fronds rossete. Stipes up to $5 \mathrm{~cm}$ long, black, scaly at the base. Laminae simple, up to $120 \mathrm{~cm}$ long or more, upto $25 \mathrm{~cm}$ wide or more, gradually narrowing towards base and apex, light green. Sori elongate along veins.

Note: This species is epiphytic fern on roadside trees or tree forest (such as Diptocarpaceae tree, Oil Palm tree (Elaeis guineensis Jack, Arecaceae)), and well known with local name "Paku Sarang or Paku Sarang Burung" that commonly used for ornamental plant. The occurrence of this species in Sumatera island, Including Riau Province had been reported in previous studies (Sofiyanti et al. 2015a,b, Pranita et al. 2017).

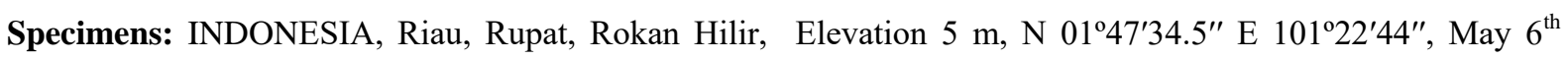
2017, Syafroni Pranata, AN11, AN12 (HR).

\section{POLYPODIACEAE}

Drynaria sparsisora (Desv.) T. Moore. Index Fil. 3481862.

(Figs. 2A-B)

Drynaria linnei Bory ex Bedd. Ferns Brit. India, pl. 3151869.

Polypodium sparsisorum Desv Ges. Naturf. Freunde Berlin Mag. Neuesten Entdeck. Gesammten Naturk. 5: 3151811.

Common. Epiphyte. Rhizome creeping, densely scale. Scale lighter brown towards the apex. Dimorphic. Sterile leaves sessile, oval, lobed $c a .20 \times 19 \mathrm{~cm}$ or more. Fertile leaves with pinnatifid laminae, oblong, light green, lobed, up to $100 \mathrm{~cm}$ long and $40 \mathrm{~cm}$ wide. Sori round, sparsely distributed. 
Note: We observed that this species found Oil Palm tree (Elaeis guineensis Jack, Arecaceae). However, we also recorded the occurrence on other Dipterocarpaceae species.

Specimens: INDONESIA, Riau, Siak, Elevation 5 m, N 0128'24" E $101^{\circ} 57^{\prime 2} 27^{\prime \prime}$, July $5^{\text {th }} 2017$, Nery Sofiyanti, DS1 (HR); Riau, Bagan, Rokan Hilir, May $6^{\text {th }}$ 2017, Syafroni Pranata, Elevation 5 m, N 01 $47^{\prime} 07^{\prime \prime}$ E $101^{\circ} 22^{\prime} 26^{\prime \prime}$, AN11, AN12 (HR).
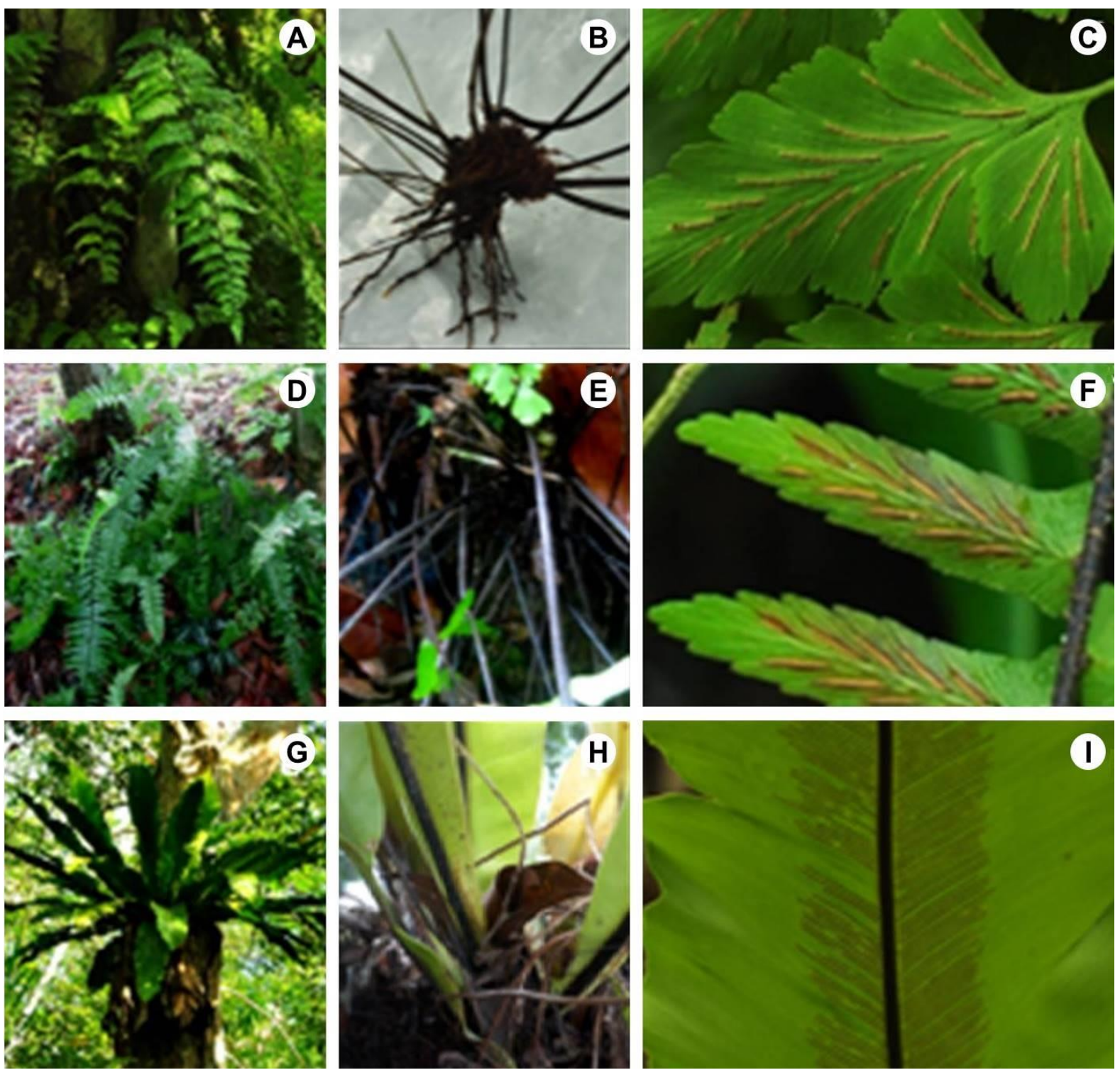

Figure 1. Aspleniaceae species from coastal regions of Riau (Left- Habitus, Center- Rhizome and base of frond, RightMature leaves showing sori): A-C, Asplenium glaucophylum; D-F, Asplenium longissimum; G-I, Asplenium nidus. [Scale bars: $\mathrm{F}=1 \mathrm{~cm} ; \mathrm{C}=2 \mathrm{~cm} ; \mathrm{B}, \mathrm{E} \& \mathrm{H}=5 \mathrm{~cm} ; \mathrm{A}, \mathrm{D}=10 \mathrm{~cm} ; \mathrm{G} \& \mathrm{I}=20 \mathrm{~cm}]$

Microsorum punctatum (L.) Copel. Univ. Calif. Publ. Bot. 16(2): 1111929.

(Figs. 2C-D)

Acrostichum punctatum L. f. Suppl. Pl. n. 4441781.

Microsorum sessile Fée. Mém. Foug. 5: 2681852.

Phymatodes polycarpus (Cav.) C. Presl. 198, t. 8, f. 191836.

Polypodium millisorum Baker. J. Linn. Soc., Bot. 15(83): 1091877.

Common. Epiphyte. Rhizome creeping. Fronds closely spaced on the rhizome, single. Stipes dark brown. Laminae lanceolate, light green, apex acute, up to 100 long or more and $15 \mathrm{~cm}$ wide, midrib rise, wavy margin. Sori numerous, round, small.

Note: We observed that this species occurs on Rambutan tree (Nephelium lappaceum L., Sapindaceae) and Oil Palm tree (Elaeis guineensis Jack, Arecaceae).

Specimens: INDONESIA, Riau, Halang, Rokan Hilir, May $6^{\text {th }} 2017$, Syafroni Pranata, Elevation $5 \mathrm{~m}, \mathrm{~N}$ 01 ${ }^{\circ} 47^{\prime} 07^{\prime \prime}$ E $101^{\circ} 22^{\prime} 26^{\prime \prime}$, MPI1 (HR). 

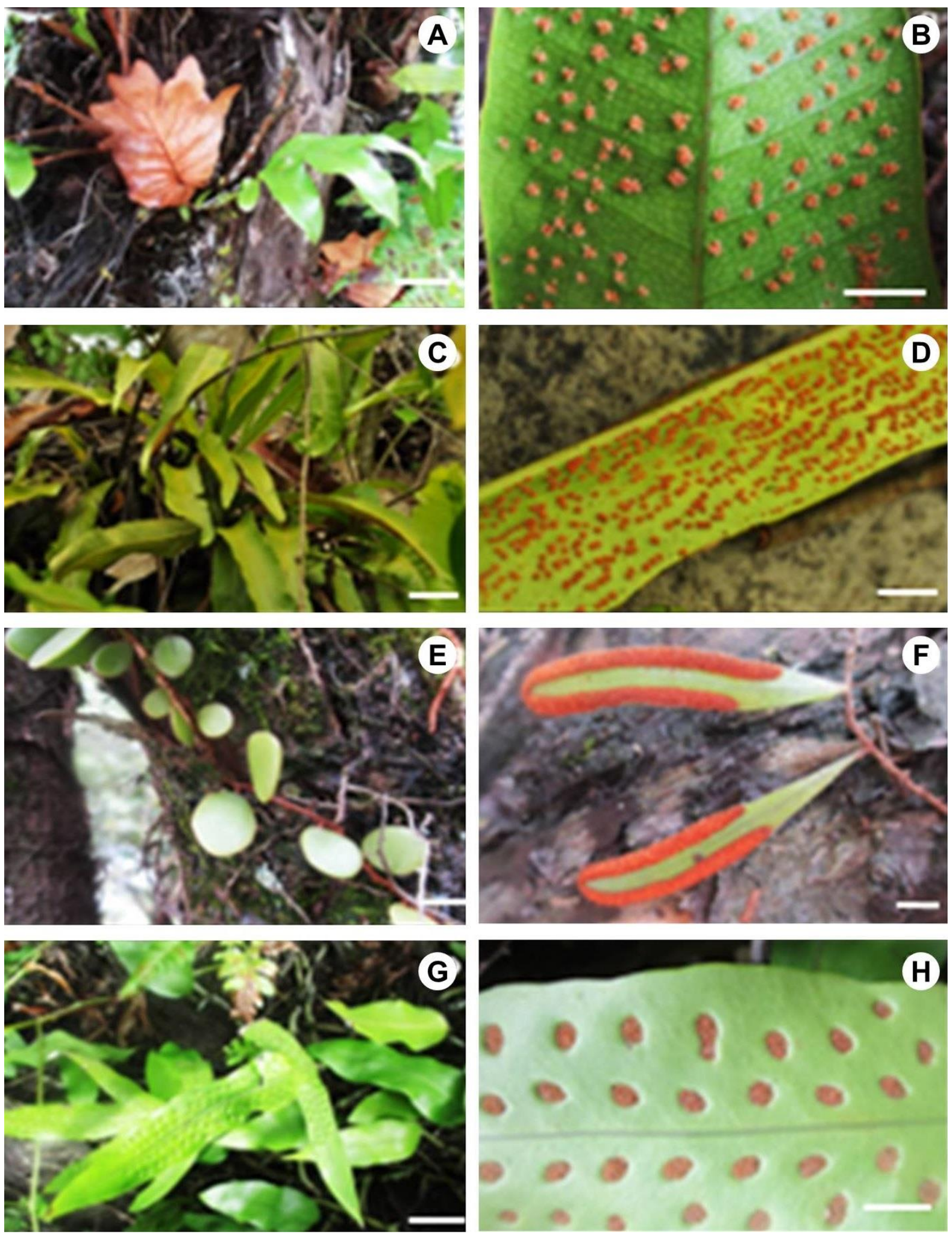

Figure 2. Polypodiaceae species from Riau coastal area (Left- Habitus, Right- Mature leaves showing sori): A-B, Drynaria sparsisora; C-D, Microsorum punctatum; E-F, Pyrrosia piloselloides; G-H, Phymatosorus scolopendria. [Scale bars: A, C $\& \mathrm{G}=5 \mathrm{~cm} ; \mathrm{B}, \mathrm{D}, \mathrm{E}, \mathrm{F} \& \mathrm{H}=1 \mathrm{~cm}]$

Pyrrosia piloselloides (L). M.G. Price.

(Figs. 2 E-F)

Common. Epiphyte. Rhizome long, creeping $c a .0 .5 \mathrm{~cm}$ diameter. Dimorphic. Frond simple. Stipes short. Sterile laminae almost circular to oval, light green, $c a .1 \times 1.5 \mathrm{~cm}$ or more, margin entire, fleshy. Fertile laminae lanceolate, $c a .15 \mathrm{~cm} \times 0.8 \mathrm{~cm}$ wide, apex rounded. Sori elongated forming marginal line.

Note: Occur on many tree species, i.e. Cemara (Thuja occidentalis L, Cupressaceae), Coconut (Cocos nucifera L., Arecaceae), Mango tree (Mangifera indica L., Anacardiaceae), Oil Palm tree (Elaeis guineensis Jack, 
Arecaceae) and Rambutan tree (Nephelium lappaceum L, Sapindaceae). The occurrence of this species in Riau had been recorded by Sofiyanti et al. 2015a,b at conservation forest Taman Hutan Raya Sultan Syarif Hasyim.

Specimens: INDONESIA, Riau, Bengkalis, July $6^{\text {th }} 2017$, Nery Sofiyanti, Elevation 5 m, N 01 $33^{\prime} 26^{\prime \prime}$ E $102^{\circ} 18^{\prime} 19^{\prime \prime}$, PPI1, PPI2 (HR).

Phymatosorus scolopendria (Burm. F.) Pic. Serm.

(Figs. 2G-H)

Chrysopteris phymatodes (L.) Link. Fil. Spec. 1221841.

Microsorum scolopendria (Burm. f.) Copel. Univ. Calif. Publ. Bot. 16(2): 1121929.

Phymatodes scolopendria (Burm. f.) Ching. Contr. Inst. Bot. Natl. Acad. Peiping 2(3): 631933.

Polypodium phymatodes L. Mant. Pl. 3061771.

Common. Terrestrial. Rhizome long creeping, light green, scaly. Scale dark. Stipes up to $40 \mathrm{~cm}$ or more at maturity. Dimorphic. Laminae oblong to ovate, subdeltoid, simple or pinnatifid, $c a .40 \mathrm{~cm}$ long or more. Sori round to oval, sparsely distributed on the lower surface.

Note: As epiphytic fern on Oil Palm tree (Elaeis guineensis Jack, Arecaceae). Traditionally used for treating ulcer, cough, rheumatic and skin wound. Local name: Paku Sisik Naga, Paku Duduwitan, Picisan.

Specimens: INDONESIA, Riau, Bengkalis, July $6^{\text {th }} 2017$, Nery Sofiyanti, Elevation 5 m, N 01 $26^{\prime} 54^{\prime \prime}$ E $101^{\circ} 10^{\prime} 54^{\prime \prime}$, PSO1, PSO2 (HR); Riau, Halang, May 6 ${ }^{\text {th }} 2017$, Syafroni Pranata, Elevation 5 m, N 01 $47^{\prime} 54^{\prime \prime}$ E $101^{\circ} 23^{\prime} 50^{\prime \prime}$, PSO3, PSO4 (HR).

\section{Identification key}

A key to identify the species of Aspleniaceae and Polypodiaceae families, specifically for the coastal regions of Riau, Indonesia has been provide here,

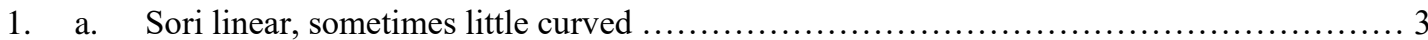

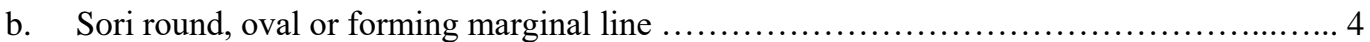

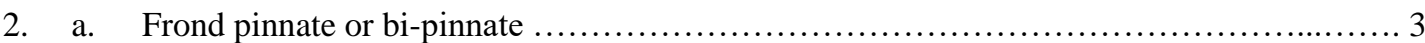

b. Fronds simple, rossete, sori linear .................................. Asplenium nidus

3. a. Fronds pinnatifid, pinnae lancet, sori $c a$. 10 each pinna, bulbil present

Asplenium longissimum

b. Fronds bipinnatifid almost triangular, sori $c a$. 20 per pinnula, bulbil absent

Asplenium glaucophylum

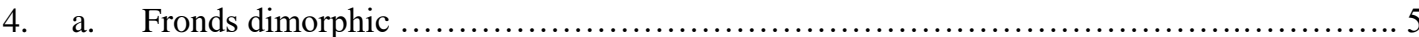

b. Fronds monomorphic ..................................... Microsorum punctatum

5. a. Fertile laminae up to $40 \mathrm{~cm}$ long or more, margin lobed .............................. 6

b. Fertile laminae ca. $20 \mathrm{~cm}$, margin entire, fleshy .................... Pyrrosia piloselloides

6. a. Sterile fronds sessile, sori pale yellow............................ Drynaria sparsisora

b. Sterile fronds closed spaced, sori dark brown and clearly present.

Phymatosorus scolopendria

Palynological study

Table 1. Spore characteristics of examined species.

\begin{tabular}{|c|c|c|c|c|c|c|c|}
\hline Species & $\begin{array}{l}\text { Spore } \\
\text { grains }\end{array}$ & $\begin{array}{l}\text { Spore } \\
\text { type }\end{array}$ & $\begin{array}{l}\text { Surface } \\
\text { pattern }\end{array}$ & $\begin{array}{r}\mathbf{P} / \mathbf{E} \\
\text { ratio }\end{array}$ & Shape class & Spore size $(\mu \mathrm{m})$ & $\begin{array}{r}\text { Size } \\
\text { Class } \\
\end{array}$ \\
\hline \multicolumn{8}{|l|}{ Aspleniaceae } \\
\hline $\mathrm{AG}$ & Monad & Monolete & Rugulate & 0.64 & Oblate & $40.05 \pm 5.50$ & Medium \\
\hline AL & Monad & Monolete & Costate & 0.58 & Oblate & $48.67 \pm 1.33$ & Medium \\
\hline $\mathrm{AN}$ & Monad & Monolete & Lophate & 0.76 & Subspheroidal & $33.90 \pm 2.10$ & Medium \\
\hline \multicolumn{8}{|l|}{ Polypodiaceae } \\
\hline $\mathrm{DS}$ & Monad & Monolete & Baculate & 0.43 & Peroblate & $36.39 \pm 2.20$ & Medium \\
\hline MP & Monad & Monolete & Psilate & 0.62 & Oblate & $54.20 \pm 5.20$ & Large \\
\hline PS & Monad & Monolete & Rugulate & 0.61 & Oblate & $50.57 \pm 6.35$ & Large \\
\hline PP & Monad & Monolete & $\begin{array}{l}\text { Scabrate } \\
\text { echinate }\end{array}$ & 0.56 & Oblate & $57.88 \pm 2.12$ & Large \\
\hline
\end{tabular}

Note: P- Polar axis; E- Equatorial axis; AG- Asplenium glaucophylum; AL- Asplenium longissimu, AN- Asplenium nidus; DS- Drynaria sparsisora; MP- Microsorum punctatum; PH- Phymatosorus sclopendria; PP- Pyrrosia piloselloides.

The palynology term comes from Greek "to sprinkle" that resembles pollen or spore that sprinkled as dust (Vernal 2014). The pollen and spore characteristic plays an important role in plant identification (Sofiyanti \& www.tropicalplantresearch.com 
Yen 2010), classification (Talip et al. 2017) as well as other systematics works (Vernal 2014). The spore study of Aspleniaceae members had been reported on Asplenium from India (Nayar \& Devi 1964), Cuba (Regalado \& Sanchez 2002), Saudi Arabia (Lashin 2012), China (Wei \& Dong 2012) and Java (Indonesia) (Pranita et al. 2017). While spore studies of Polypodiaceae had been reported on Genera Phymatodes, Microsorium, Crypsonus, Lepisorus and Campyloneurum from India (Pal \& Pal 1970). Morbelli \& Giudice (2010) also reported the spore morphology of Polypodiaceae, genera Campyloneurum, Microgramma, Pecluma, Phlebodium, Pleopeltis and Serpocaulum from Argentina. The spore morphology of Aspleniaceae and Polypodiaceae species examined in this study has been shown in figure 3 and 4 respectively. On the other hand the spore characteristics of each examined species have been shown in table 1.
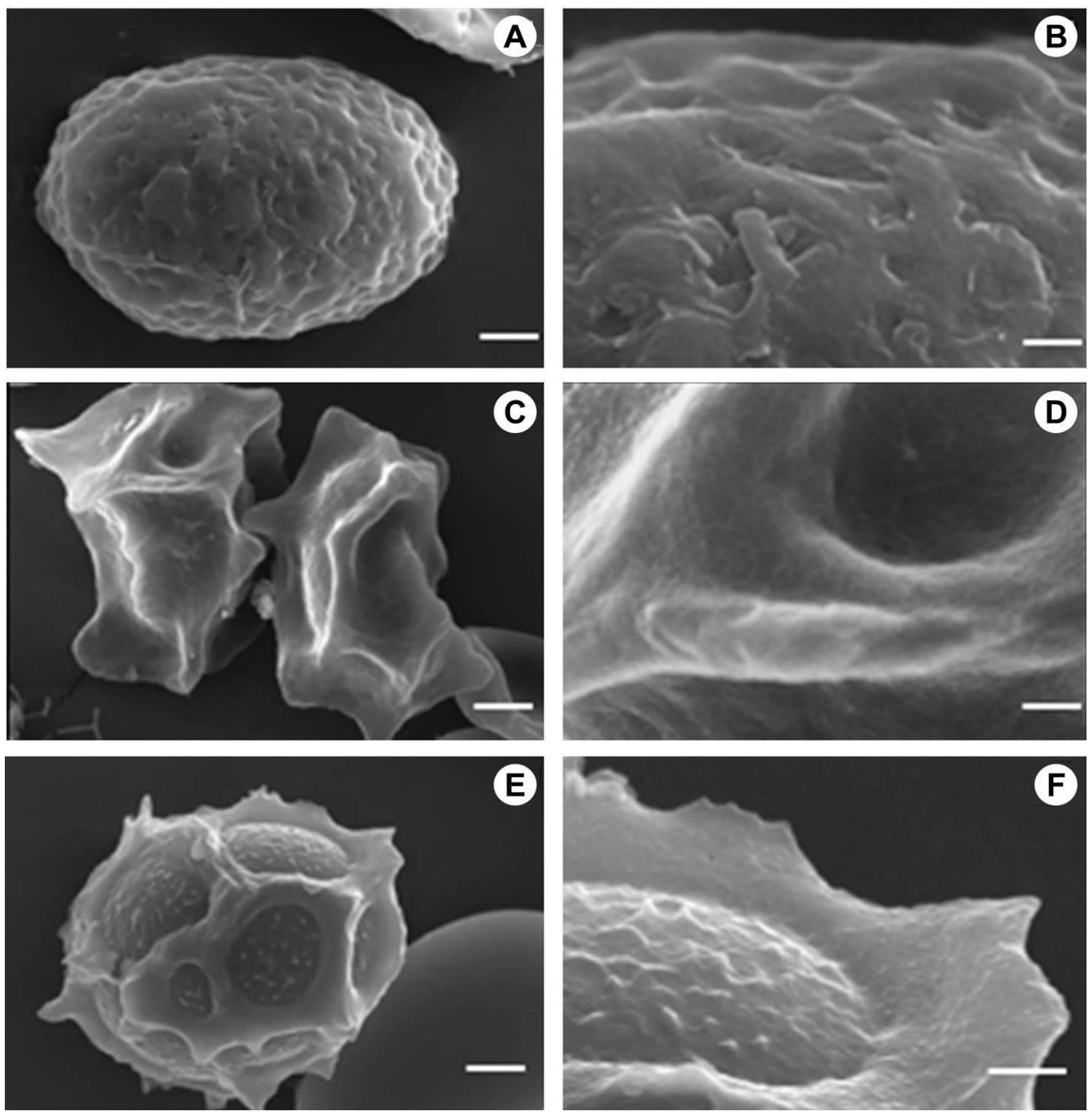

Figure 3. Spore feature of Aspleniaceae species: A-B, Asplenium glaucophylum; C-D, Asplenium longissimum; E-F, Asplenium nidus. [Scale bars: A, C \& $\mathrm{E}=5 \mu \mathrm{m} ; \mathrm{B}, \mathrm{D}, \mathrm{F}=2 \mu \mathrm{m}$ ]

\section{General type and size of spore}

In this study, we observed monad spore grain from all of the examined species. Monad spore grain is not attached each other but release in single grain (Sofiyanti \& Yen 2010). The observation of spore features also show the similar basic spore type for all of the species examined, i.e. monolete spore. This type is found in all of Aspleniaceae and Polypodiaceae species. Monolete spore is one of basic type of fern spore that characterized by its bean-shaped and has laesura in proximal part (Sofiyanti et al. 2017). The monolete spore was also reported on other Aspleniaceae species, especially from Genus Asplenium by Regalado \& Sanchez (2002), Lashin (2012), Mazooji \& Salimpour (2014) and Vijayakanth \& Sathis (2016). 

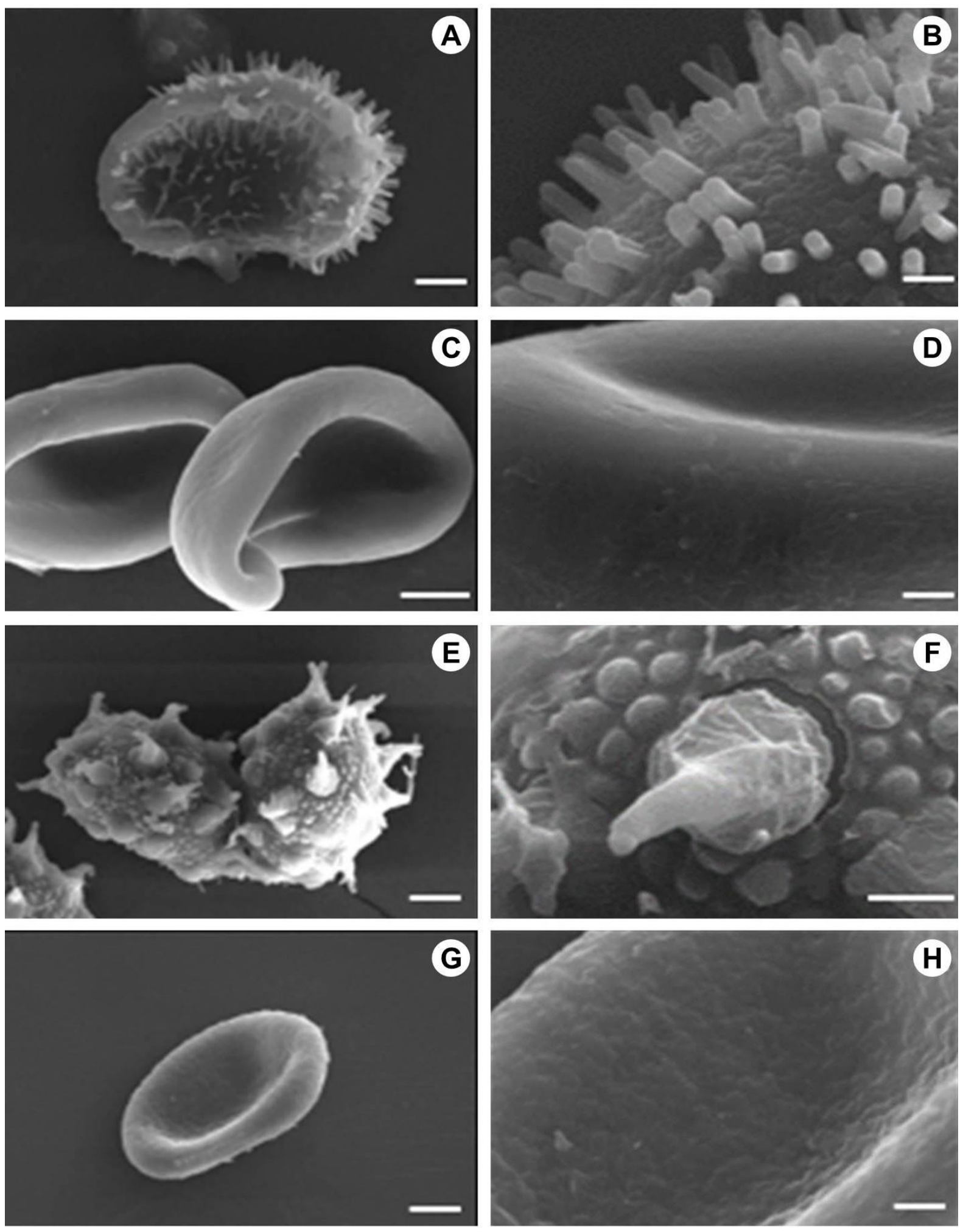

Figure 4. Spore morphology of Polypodiaceae species (Left- Spore grains, Right- Close up on spore surface): A-B, Drynaria sparsisora; $\mathbf{C}-\mathbf{D}$, Microsorum punctatum; E-F, Pyrrosia piloselloides; G-H, Phymatosorus scolopendria. [Scale bars: $\mathrm{A} \& \mathrm{~F}=5 \mu \mathrm{m} ; \mathrm{B}, \mathrm{D} \& \mathrm{H}=2 \mu \mathrm{m} ; \mathrm{C}, \mathrm{E} \& \mathrm{G}=10 \mu \mathrm{m}]$

The spore classification based on the spore size proposed by Erdtman (1957) recorded 6 types. However, we only observed two spore type observed in this study i.e. medium and large. Medium spore has the longest measurement ranged from 25 to $50 \mu \mathrm{m}$ (Erdtman 1957) and observed on all of Aspleniaceae species (Asplenium glaucophyllum, Asplenium longissimum and Asplenium nidus). This result supports the previous studies that reported the medium spore size on most of Asplenium species (Nayar \& Devi 1964, Regalado \& Sanchez 2002, Lashin 2012, Vijayakanth \& Sathish 2017). However, the large spore size (spore size range from 50 to $100 \mu \mathrm{m}$ ) also reported on few Asplenium members, such as Asplenium griffithianuni Hook (Nayar \& Devi 1964), Asplenium aethiopicum and Asplenium trichomanes (Lashin 2012).

www.tropicalplantresearch.com 
For Polypodiaceae species, one out of four examined taxa (Drynaria sparsisora) showed medium spore, while three other taxa had large spore, with 50-100 $\mu \mathrm{m}$ spore size (Erdtman 1957). The large spore type was observed from three Polypodiaceae species, Microsorum punctatum, Pyrrosia piloselloides and Phymatosorus scolopendria. The largest spore size $(57.88 \pm 2.12 \mu \mathrm{m})$ was observed on Phymatosorus scolopendria.

Spore class observed on Aspleniaceae species were oblate (Asplenium glaucophyllum and Asplenium longissimum) and suphreoidal (Asplenium nidus). Oblate spore class was also observed on three polypodiaceae species in this study (Microsorum punctatum, Phymatosorus scolopendria and Pyrrosia pilloseloides). On the other hand, Drynaria sparsisora had peroblate spore (see table 1 for detail).

\section{Surface pattern}

The surface pattern of fern spore provides taxonomical information (Regalado \& Sanchez 2002, Morbelli \& Giudice 2010). The spore surface of Asplenium in this study shows distinct pattern among the examined species i.e. rugulate (Asplenium glaucophyllum), costate (Asplenium longissimum) and lophate (Asplenium nidus). Rugulate spore surface has very low warted spore (Atri et al. 2014). Costate spore has broader base of perisphore with convex side arranged in an irregular pattern (Regalado \& Sanchez 2002), while lophate pattern has costate to cristate perispore (Lin \& Viane 2013).

The surface pattern of spores belong to Family Polypodiaceae show the variation within the species, i.e. baculate (Drynaria sparsisora), psilate (Microsorum punctatum), regulate (Phymatosorus scolopendria) dan scabrate echinat (Pyrrosia piloselloides). Figure 4 presents the spore morphologies of four Polypodiaceae species. According to Morbelli \& Giudice (2010), the spore ornamentation of Polypodiaceae varies within examined taxa.

\section{ACKNOWLEDGEMENTS}

This study was funded by Research Grant from Ministry of Research, Technology and Higher Education, Indonesia.

\section{REFERENCES}

Atri NS, Kaur M, \& Sharma S (2014) SEM study of exosporial ornamentation of Basidiospores Ingenus Russula, Lactarius and Lactifluus from North-West Himalaya. In: Proceedings of the $8^{\text {th }}$ International Conference on Mushroom Biology and Mushroom Products (ICMBMP8).

Beukema H. \& van Noordwijk M (2004) Terrestrial pteridophytes as indicators of a forest-like environment in rubber production systems in the lowlands of Jambi, Sumatra. Agriculture, Ecosystems and Environment 104: 63-73.

Erdtman G (1957) Pollen and Spore Morphology and Plant Taxonomy: Gymnospermae, Pteridophyta, Bryophyta, Illustrations. Almqvist and Wiksell. Stockholm.

Lashin GMA (2012) Palynological Studies of Some Species of Aspleniaceae-Pteridophyta. American Journal of Plant Sciences 3: 397-402.

Lin YX \& Viane R (2013) Aspleniaceae. In: Wu ZY, Raven PH \& Hong DY (eds) Flora of China, 2-3 (Pteridophytes). Science Press and St. Louis: Missouri Botanical Garden Press, Beijing, pp. 267-316.

Marpaung AA, Sofiyanti N, Iriani D \& Fitmawati (2016) Morfologi spora paku Pteridaceae di Hutan PT. CPI Rumbai Riau. Jurnal Riau Biologia 1(2): 148-154.

Mazooji A \& Salimpour F (2014) Spore Morphology of 34 Species of Monilophyta from Northern Parts of Iran. Annual Research \& Review in Biology 4(6): 924-935.

Moran RC, Hanks, JG \& Rouhan G (2007) Spore morphology in relation to phylogeny in the fern genus Elaphoglossum (Dryopteridaceae). International Journal of Plant Sciences 168(6): 905-929.

Morbelli MA \& Giudice GE (2010) Spore wall ultrastructure of Polypodiaceae from north-western Argentina. Grana 49: 204-214.

Nayar BK \& Devi S (1964) Spore Morphology of Indian Ferns II. Aspleniaceae and Blechnaceae. Grana 5(2): 222-246.

Ohlsen DJ, Perrie L, Shepherd LD, Brownsey P \& Bayly M (2014) Phylogeny of the fern family Aspleniaceae in Australasia and the south-western Pacific. Australian Systematic Botany 27: 355-371.

Pal S \& Pal N (1970) Spore Morphology and Taxonomy of Polypodiaceae. Grana 10(2): 141-148.

Piggott AG \& Piggott CJ (1988) Ferns of Malaysia in colour. Tropical Press, Kuala Lumpur.

Pranita HS, Mahanal S \& Sari MS (2017) Karakteristik Spora Tumbuhan Paku Asplenium Kawasan Hutan Raya R. Soerjo. Jurnal Pendidikan: Teori, Penelitian, Dan Pengembangan 2(4): 454-458 
Regalado L \& Sanchez C (2002) Spore morphology as a taxonomic tool in the delimitation of three Asplenium L. species complexes (Aspleniaceae: Pteridophyta) in Cuba. Grana 41(2): 107-113.

Silva AG \& Schwartsburd PB (2017) Ferns of Viçosa, Minas Gerais State, Brazil: Polypodiaceae (Polypodiales, Filicopsida, Tracheophyta). Hoehnea 44(2): 251-268.

Smith AR, Kreier HP, Haufler CH, Ranker TA \& Schneider H (2006b) Serpocaulon (Polypodiaceae), a new genus segregated from Polypodium. Taxon 55: 919-930.

Smith AR, Pryer KM, Schuettpelz E, Korall P, Schneider H \& Wolf PG (2006a) A classification for extant ferns. Taxon 55 (3): 705-731.

Sofiyanti N \& Yen CC (2010) Morphology of ovule, seed and pollen grain of Rafflesia R. Br. (Rafflesiaceae). Bangladesh Journal of Plant Taxonomy 19(2): 109-117.

Sofiyanti N (2013) The diversity of epiphytic fern on the oil palm tree (Elaeis Guineensis Jacq.) In Pekanbaru, Riau. Jurnal Biologi Udayana 17(2): 51-55.

Sofiyanti N, Iriani D \& Roza AA (2015a) Morfologi tumbuhan Paku di Taman Hutan Raya Sultan Syarif Hasyim, Riau. Unri Press, Pekanbaru.

Sofiyanti N, Iriani D, Fitmawati \& Marpaung AA (2017) Karakteritik dan Metode Pembuatan Preparat Spora Pteridoflora. UNRI Press, Pekanbaru.

Sofiyanti N, Iriani D, Fitmawati \& Roza AA (2015b) Stenochlaena riauensis (Blechnaceae), A new fern species from Riau, Indonesia. Bangladesh Journal of Plant Taxonomy 22(2): 137-141.

Sofiyanti N, Mat-Salleh K, Mahmud K, Mazlan NZ, Hasein MRA \& Burslem DFRP (2016) Rafflesia parvimaculata (Rafflesiaceae), a new species of Rafflesia from Peninsular Malaysia. Phytotaxa 253(3): 207213.

Talip N, Cutler DF, Puad AS, Ismail BS, Ruzi AR \& Juhari A (2017) Diagnostic and systematic significance of petiole anatomy in the identification of Hopea Species (Dipterocarpaceae). South African Journal of Botany 111: 111-125.

Vernal A (2014) Palynology (Pollen, Spores, etc). Encyclopedia of Marine Geosciences. [DOI 10.1007/978-94007-6644-0_87-1]

Vijayakanth P \& Sathish S (2016) Studies On The Spore Morphology Of Pteridophytes From Kolli Hills, Eastern Ghats, Tamil Nadu, India. International Journal of Research in Engineering and Bioscience 4(1): 112.

Wei L \& Dong S (2012) Taxonomic studies on Asplenium sect. Thamnopteris (Aspleniaceae) II: spore morphology. Nordic Journal of Botany 30: 90-103.

Wulandari D, Sofiyanti N \& Fitmawati (2016) Keanekaragaman Jenis Polypodiaceae Di Hutan PT. CPI Rumbai Provinsi Riau Berdasarkan Karakter Morfologi. Jurnal Riau Biologia 1(2): 135-139.

Yusna M, Sofiyanti N \& Fitmawati (2016) Keanekaragaman pteridaceae berdasarkan karakter morfologi dan fitokimia di Hutan PT. Chevron Pacific Indonesia (PT. CPI) Rumbai. Jurnal Riau Biologia 1(2): 165-172. 